\title{
SOME SYMMETRIC PROPERTIES OF KENMOTSU MANIFOLDS ADMITTING SEMI-SYMMETRIC METRIC CONNECTION
}

\author{
Venkatesha Venkatesh, Arasaiah Arasaiah, \\ Vishnuvardhana Srivaishnava Vasudeva \\ and Naveen Kumar Rahuthanahalli Thimmegowda
}

\begin{abstract}
The objective of the present paper is to study some symmetric properties of the Kenmotsu manifold endowed with a semi-symmetric metric connection. Here we consider pseudo-symmetric, Ricci pseudo-symmetric, projective pseudo-symmetric and $\phi$-projective semi-symmetric Kenmotsu manifolds with respect to the semi-symmetric metric connection. Finally, we provide an example of the 3-dimensional Kenmotsu manifold admitting a semi-symmetric metric connection which verifies our result.

Keywords: Kenmotsu manifold; projective curvature tensor; semi-symmetric metric connection; $\eta$-Einstein manifold.
\end{abstract}

\section{Introduction}

In 1932, Hayden [12] introduced the idea of metric connection with a torsion on a Riemannian manifold. By considering the torsion tensor of a linear connection, Friedmann and Schouten [11] gave a new connection called semi-symmetric connection. The torsion tensor with respect to the semi-symmetric connection $\bar{\nabla}$ is given by

$$
\bar{T}(X, Y)=\bar{\nabla}_{X} Y-\bar{\nabla}_{Y} X-[X, Y] .
$$

The connection $\bar{\nabla}$ is called a semi-symmetric metric connection [12] if $\bar{\nabla} g=0$, otherwise, non-metric connection. A relation between the semi-symmetric metric connection $\bar{\nabla}$ and the Levi-Civita connection $\nabla$ on $(M, g)$ established by Yano [18] is given by

$$
\bar{\nabla}_{X} Y=\nabla_{X} Y+\eta(Y) X-g(X, Y) \xi .
$$

Semi-symmetric manifolds form a subclass of the class of pseudo-symmetric manifolds. The concept of pseudo-symmetric manifold was introduced by Chaki and

Received November 28, 2017; accepted December 19, 2018

2010 Mathematics Subject Classification. Primary 53C05; Secondary 53C20, 53C50

* corresponding author 
Chaki [8] and Deszcz [10] in two different ways. Here we study the properties of pseudo-symmetric manifolds with a semi-symmetric metric connection in the Deszcz sense. An $n$-dimensional Riemannian manifold $M$ is called pseudo-symmetric in the sense of Deszcz [10] if the Riemannian curvature tensor R satisfies the following relation

$$
(R(X, Y) \cdot R)(U, V) W=L_{R}\left(\left(X \wedge_{g} Y\right) \cdot R\right)(U, V) W
$$

for all the vector fields $X, Y, Z, U, V, W \in T M$. Where $L_{R}$ is a smooth function on $M$ and $X \wedge_{g} Y$ is an endomorphism defined by

$$
\left(X \wedge_{g} Y\right) Z=g(Y, Z) X-g(X, Z) Y .
$$

The notion of semi-symmetric metric connection has been weakened by many geometers such as $[2,3,5,9,15,17]$ etc., with different structures of manifolds and submanifolds. In particular, De [1] and Bagewadi et. al. [4] studied semisymmetric metric connection on Kenmotsu manifolds with a projective curvature tensor. Also in [16], Singh et. al. studied the semi-symmetric metric connection in an $\epsilon$-Kenmotsu manifold.

The projective curvature tensor $\bar{P}$ with respect to the semi-symmetric metric connection on a Kenmotsu manifold is defined by [1]

$$
\bar{P}(X, Y) Z=\bar{R}(X, Y) Z-\frac{1}{n-1}[\bar{S}(Y, Z) X-\bar{S}(X, Y) Z],
$$

for $X, Y, Z \in \chi(M)$. Here $\bar{S}$ is the Ricci tensor with respect to the semi-symmetric metric connection.

Further, a relation between the curvature tensor $\bar{R}$ of the semi-symmetric metric connection $\bar{\nabla}$ and the curvature tensor $R$ of the Levi-Civita connection $\nabla$ is given by $[18]$

$$
\begin{aligned}
\bar{R}(X, Y) Z & =R(X, Y) Z-\alpha(Y, Z) X+\alpha(X, Z) Y \\
& -g(Y, Z) L X+g(X, Z) L Y
\end{aligned}
$$

where $\alpha$ is a tensor field of type $(0,2)$ and $L$ is a tensor field of type $(1,1)$ which is given by

$$
\alpha(Y, Z)=g(L Y, Z)=\left(\nabla_{Y} \eta\right)(Z)-\eta(Y) \eta(Z)+\frac{1}{2} \eta(\xi) g(Y, Z),
$$

for any vector fields $X, Y, Z \in \chi(M)$. From (1.6), it follows that

$$
\bar{S}(Y, Z)=S(Y, Z)-(n-2) \alpha(Y, Z)-a g(Y, Z),
$$

where $\bar{S}$ denotes the Ricci tensor with respect to $\bar{\nabla}$ and $a=$ trace of $\alpha$.

Motivated by these studies, we investigate the semi-symmetric metric connection due to Yano [18] on Kenmotsu manifolds. The paper is organized as follows. After giving preliminaries and basic results of the Kenmotsu manifold in Section 
2 , in Section 3 we study pseudo-symmetric Kenmotsu manifolds with respect to the semi-symmetric metric connection, proving that either $L_{\bar{R}}=-2$ or the manifold is $\eta$-Einstein. In the next section we prove that in a Ricci pseudo-symmetric Kenmotsu manifold with respect to the semi-symmetric metric connection, either $L_{\bar{S}}=-2$ or the manifold is $\eta$-Einstein. Sections 5 and 6 are devoted to the study of projective pseudo-symmetric and $\phi$-projective semi-symmetric Kenmotsu manifolds with respect to the semi-symmetric metric connection. Finally, we construct an example of a 3-dimensional Kenmotsu manifold admitting the semi-symmetric metric connection and verify the results.

\section{Preliminaries}

Let $M$ be an $n$-dimensional almost contact Riemannian manifold equipped with the almost contact metric structure $(\phi, \xi, \eta, g)$, where $\phi$ is a $(1,1)$ tensor field, $\xi$ is a characteristic vector field, $\eta$ is a 1-form and $g$ is the Riemannian metric satisfying the following conditions [7];

(2.1) $\phi^{2}(X)=-X+\eta(X) \xi, \quad \eta(\xi)=1, \quad \eta \circ \phi=0, \quad \phi \xi=0, \quad g(X, \xi)=\eta(X)$,

(2.2) $g(\phi X, \phi Y)=g(X, Y)-\eta(X) \eta(Y)$,

for all vector fields $X, Y$ on $M$. If an almost contact metric manifold satisfies

$$
\left(\nabla_{X} \phi\right)(Y)=g(\phi X, Y) \xi-\eta(Y) \phi X,
$$

then $M$ is called a Kenmotsu manifold [14]. Here $\nabla$ denotes the operator of covariant differentiation with respect to $g$. From (2.3), it follows that

$$
\begin{aligned}
& \nabla_{X} \xi=X-\eta(X) \xi, \\
& \left(\nabla_{X} \eta\right)(Y)=g(X, Y)-\eta(X) \eta(Y) .
\end{aligned}
$$

In a Kenmotsu manifold $M$, the following relations hold:

(2.6) $\eta(R(X, Y) Z)=[g(X, Z) \eta(Y)-g(Y, Z) \eta(X)]$,

(2.7) (a) $R(\xi, X) Y=[\eta(Y) X-g(X, Y) \xi]$, (b) $R(X, Y) \xi=[\eta(X) Y-\eta(Y) X]$,

(2.8) (a) $S(X, Y)=-(n-1) g(X, Y),(b) Q X=-(n-1) X$,

(2.9) (a) $S(X, \xi)=-(n-1) \eta(X),(b) S(\xi, \xi)=-(n-1),(c) Q \xi=-(n-1) \xi$,

(2.10) $\left(\nabla_{W} R\right)(X, Y) \xi=g(W, X) Y-g(W, Y) X-R(X, Y) W$,

(2.11) $S(\phi X, \phi Y)=S(X, Y)+(n-1) \eta(X) \eta(Y)$.

Now by using (1.7), (2.1) and (2.5) in (1.6), we have the following relation

$$
\begin{aligned}
\bar{R}(X, Y) Z & =R(X, Y) Z-3[g(Y, Z) X-g(X, Z) Y]+2[\eta(Y) X \\
& -\eta(X) Y] \eta(Z)+2[g(Y, Z) \eta(X)-g(X, Z) \eta(Y)] \xi
\end{aligned}
$$

Contracting $X$ in (2.12), we get

$$
\bar{S}(Y, Z)=S(Y, Z)-(3 n-5) g(Y, Z)+2(n-2) \eta(Y) \eta(Z) .
$$


Again contracting $Y$ and $Z$ in (2.13), we get

$$
\bar{r}=r-(n-1)(3 n-4),
$$

where $\bar{r}$ and $r$ are the scalar curvatures with respect to the semi-symmetric metric connection and the Levi-Civita connection respectively.

\section{Pseudo-symmetric Kenmotsu manifold with respect to the semi-symmetric metric connection}

Definition: An $n$-dimensional Kenmotsu manifold $M$ is said to be pseudosymmetric with respect to semi-symmetric metric connection if the curvature tensor $\bar{R}$ of $\bar{\nabla}$ satisfies the condition

$$
(\bar{R}(X, Y) \cdot \bar{R})(U, V) W=L_{\bar{R}}\left(\left(X \wedge_{g} Y\right) \cdot \bar{R}\right)(U, V) W
$$

where $L_{\bar{R}}$ is a function on $M$. From (3.1), we have

$$
\begin{array}{ll} 
& \bar{R}(X, Y)(\bar{R}(U, V) W)-\bar{R}(\bar{R}(X, Y) U, V) W-\bar{R}(U, \bar{R}(X, Y) V) W \\
& -\bar{R}(U, V)(\bar{R}(X, Y) W)=L_{\bar{R}}\left[\left(X \wedge_{g} Y\right)(\bar{R}(U, V) W)-\bar{R}\left(\left(X \wedge_{g} Y\right) U, V\right) W\right. \\
(3.2) \quad & \left.-\bar{R}\left(U,\left(X \wedge_{g} Y\right) V\right) W-\bar{R}(U, V)\left(X \wedge_{g} Y\right) W\right] .
\end{array}
$$

Replacing $X$ by $\xi$ in (3.2), we get

$$
\begin{aligned}
& \bar{R}(\xi, Y)(\bar{R}(U, V) W)-\bar{R}(\bar{R}(\xi, Y) U, V) W-\bar{R}(U, \bar{R}(\xi, Y) V) W \\
& -\bar{R}(U, V)(\bar{R}(\xi, Y) W)=L_{\bar{R}}\left[\left(\xi \wedge_{g} Y\right)(\bar{R}(U, V) W)-\bar{R}\left(\left(\xi \wedge_{g} Y\right) U, V\right) W\right. \\
& \left.-\bar{R}\left(U,\left(\xi \wedge_{g} Y\right) V\right) W-\bar{R}(U, V)\left(\xi \wedge_{g} Y\right) W\right] .
\end{aligned}
$$

Using (1.4) and (2.12) in (3.3) and then taking the inner product with $\xi$, we obtain

$$
\begin{aligned}
& \left(L_{\bar{R}}+2\right)[-\bar{R}(U, V, W, Y)+\eta(\bar{R}(U, V) W) \eta(Y)+2 g(Y, U) \eta(V) \eta(W) \\
& -2 g(Y, U) g(V, W)-\eta(\bar{R}(Y, V) W) \eta(U)-2 g(Y, V) \eta(U) \eta(W) \\
& +2 g(Y, V) g(U, W)-\eta(\bar{R}(U, Y) W) \eta(V)-\eta(\bar{R}(U, V) Y) \eta(W)]=0 .
\end{aligned}
$$

On plugging $U=Y=e_{i}$ in (3.4) and taking summation over $i$, we get

$$
\left(L_{\bar{R}}+2\right)[S(V, W)-(n-5) g(V, W)+2(n-1) \eta(V) \eta(W)]=0 .
$$

This implies that either $L_{\bar{R}}=-2$ or

$$
S(V, W)=(n-5) g(V, W)+2(1-n) \eta(V) \eta(W) .
$$

On contracting (3.6), we get

$$
r=n(n-7)+2 .
$$

Hence we can state the following:

Theorem 3.1. Let $M$ be an $n$-dimensional pseudo-symmetric Kenmotsu manifold with respect to semi-symmetric metric connection. Then either $L_{\bar{R}}=-2$ or the manifold is $\eta$-Einstein with constant scalar curvature $r=n(n-7)+2$ with respect to Levi-Civita connection. 


\section{Ricci pseudo-symmetric Kenmotsu manifold with respect to the} semi-symmetric metric connection

Definition: An $n$-dimensional Kenmotsu manifold $M$ is said to be Ricci pseudosymmetric with respect to semi-symmetric metric connection, if

$$
(\bar{R}(X, Y) \cdot \bar{S})(Z, U)=L_{\bar{S}} Q(g, \bar{S})(Z, U ; X, Y)
$$

holds true on $M$, where $L_{\bar{S}}$ is some function and $Q(g, S)$ is the Tachibana tensor on $M$. From (4.1), it follows that

$$
\begin{aligned}
& \bar{S}(\bar{R}(X, Y) Z, U)+\bar{S}(Z, \bar{R}(X, Y) U) \\
& =L_{\bar{S}}\left[\bar{S}\left(\left(X \wedge_{g} Y\right) Z, U\right)+\bar{S}\left(Z,\left(X \wedge_{g} Y\right) U\right)\right] .
\end{aligned}
$$

Putting $Y=U=\xi$ in (4.2), we have

(4.3) $\bar{S}(\bar{R}(X, \xi) Z, \xi)+\bar{S}(Z, \bar{R}(X, \xi) \xi)=L_{\bar{S}}[\bar{S}((X \wedge \xi) Z, \xi)+\bar{S}(Z,(X \wedge \xi) \xi)]$

Using (1.4), (2.12), (2.13) and (2.7) in (4.3), we can get

$$
\left(L_{\bar{S}}+2\right)[S(X, Z)-(n-3) g(X, Z)+2(n-2) \eta(X) \eta(Z)]=0 .
$$

This implies that either $L_{\bar{S}}=-2$ or

$$
S(X, Z)=(n-3) g(X, Z)+2(2-n) \eta(X) \eta(Z) .
$$

On contracting (4.5) over $X$ and $Z$, we get

$$
r=(n-1)(n-4) \text {. }
$$

Thus we can state the following theorem:

Theorem 4.1. If a Kenmotsu manifold $M$ is Ricci pseudo-symmetric with respect to semi-symmetric metric connection, then either $L_{\bar{S}}=-2$ or the manifold is $\eta$ Einstein with constant scalar curvature $r=(n-1)(n-4)$ with respect to Levi-Civita connection.

\section{Projective pseudo-symmetric Kenmotsu manifold with respect to the semi-symmetric metric connection}

Definition: An $n$-dimensional Kenmotsu manifold $M$ is said to be projective pseudo-symmetric with respect to semi-symmetric metric connection if

$$
(\bar{R}(X, Y) \cdot \bar{P})(U, V) W=L_{\bar{P}}\left(\left(X \wedge_{g} Y\right) \cdot \bar{P}\right)(U, V) W
$$

holds on $M$. Putting $Y=W=\xi$ in (5.1), we get

$$
(\bar{R}(X, \xi) \cdot \bar{P})(U, V) \xi=L_{\bar{P}}\left(\left(X \wedge_{g} \xi\right) \cdot \bar{P}\right)(U, V) \xi .
$$


Now right hand side of (5.2) can be written as

$$
\begin{aligned}
L_{\bar{P}}\left(\left(X \wedge_{g} \xi\right) \cdot \bar{P}\right)(U, V) \xi & =L_{\bar{P}}\left[\left(\left(X \wedge_{g} \xi\right) \bar{P}\right)(U, V) \xi-\bar{P}\left(\left(X \wedge_{g} \xi\right) U, V\right) \xi\right. \\
& \left.-\bar{P}\left(U,\left(X \wedge_{g} \xi\right) V\right) \xi-\bar{P}(U, V)\left(X \wedge_{g} \xi\right) \xi\right] .
\end{aligned}
$$

By virtue of (1.4), (1.5), (2.12), (2.13) and (2.7) in (5.3), we obtain

$$
L_{\bar{P}}\left(\left(X \wedge_{g} \xi\right) \cdot \bar{P}\right)(U, V) \xi=-L_{\bar{P}} \cdot \bar{P}(U, V) X .
$$

Next by considering left hand side of (5.2), we have

$$
\begin{aligned}
(\bar{R}(X, \xi) \cdot \bar{P})(U, V) \xi & =\bar{R}(X, \xi) \bar{P}(U, V) \xi-\bar{P}(\bar{R}(X, \xi) U, V) \xi \\
& -\bar{P}(U, \bar{R}(X, \xi) V) \xi-\bar{P}(U, V) \bar{R}(X, \xi) \xi
\end{aligned}
$$

Again using (1.5), (2.12), (2.13) and (2.7) in (5.5), we get

$$
(\bar{R}(X, \xi) \cdot \bar{P})(U, V) \xi=2 \bar{P}(U, V) X .
$$

Substituting (5.4) and (5.6) in (5.2), we obtain

$$
\left(L_{\bar{P}}+2\right) \bar{P}(U, V) X=0 .
$$

This leads us to the following:

Theorem 5.1. If an $n$-dimensional Kenmotsu manifold is projective pseudo-symmetric with respect to the semi-symmetric metric connection, then either $L_{\bar{P}}=-2$ or the manifold is projectively flat.

Also, in a Kenmotsu manifold, Bagewadi, Prakasha and Venkatesha [4] proved the following:

Lemma 5.1. [4] If the projective curvature tensor of a Kenmotsu manifold $M$ admitting the semi-symmetric metric connection vanishes, then $M$ reduces to an Einstein manifold with the constant scalar curvature $-n(n-1)$.

Hence from Theorem 5.1. and Lemma 5.1., we conclude that:

Corollary 5.1. A projective pseudo-symmetric Kenmotsu manifold admitting the semi-symmetric metric connection is an Einstein manifold with the constant scalar curvature with respect to the Levi-Civita connection provided $L_{\bar{P}} \neq-2$.

\section{6. $\phi$-projective semi-symmetric Kenmotsu manifold with respect to the semi-symmetric metric connection}

Definition: An $n$-dimensional Kenmotsu manifold $M$ is said to be $\phi$-projectively semi-symmetric with respect to the semi-symmetric metric connection if $\bar{P}(X, Y)$. $\phi=0$.

Let us consider an $n$-dimensional Kenmotsu manifold $M$ which is $\phi$-projective semi-symmetric. Then we have

$$
\bar{P}(X, Y) \phi Z-\phi \bar{P}(X, Y) Z=0,
$$


for any vector fields $X, Y$ and $Z$ on $M$.

By virtue of (1.5) in (6.1) gives

$$
\begin{aligned}
& \bar{R}(X, Y) \phi Z-\phi \bar{R}(X, Y) Z+\frac{1}{n-1}[\bar{S}(Y, \phi Z) X \\
& -\bar{S}(X, \phi Z) Y+\bar{S}(Y, Z) \phi X-\bar{S}(X, Z) \phi Y]=0 .
\end{aligned}
$$

On plugging $Y=\xi$ in (6.2) and then using (2.12), (2.13) and (2.7), we obtain

$$
2 g(X, \phi Z) \xi-\frac{1}{n-1} \bar{S}(X, \phi Z) \xi=0 .
$$

Now taking the inner product of the above equation with $\xi$, we get

$$
2 g(X, \phi Z)-\frac{1}{n-1} \bar{S}(X, \phi Z)=0 .
$$

Replacing $Z$ by $\phi Z$ in (6.4) and then by virtue of (2.1) and (2.13), we obtain

$$
S(X, Z)=A g(X, Z)+B \eta(X) \eta(Z),
$$

where $A=5 n-7$ and $B=-2(3 n-5)$.

Hence we can state the following:

Theorem 6.1. An $n$-dimensional $\phi$-projective semi-symmetric Kenmotsu manifold with respect to the semi-symmetric metric connection is $\eta$-Einstein with respect to the Levi-Civita connection.

\section{Example}

Consider a 3-dimensional manifold $M=\left\{(x, y, z) \in \mathbb{R}^{3}: z \neq 0\right\}$, where $(x, y, z)$ are the standard coordinates in $\mathbb{R}^{3}$. We choose the vector fields

$$
E_{1}=-e^{-z} \frac{\partial}{\partial x}, \quad E_{2}=e^{-z} \frac{\partial}{\partial y}, \quad E_{3}=\frac{\partial}{\partial z},
$$

which are linearly independent at each point of $M$. Let $g$ be the Riemannian metric defined by

$$
g=e^{2 z}(d x \otimes d x+d y \otimes d y)+\eta \otimes \eta,
$$

where $\eta$ is the 1 -form defined by $\eta(X)=g\left(X, E_{3}\right)$, for any vector field $X$ on $M$. Then $\left\{E_{1}, E_{2}, E_{3}\right\}$ is an orthonormal basis of $M$. We define a $(1,1)$ tensor field $\phi$ as

$$
\phi\left(X \frac{\partial}{\partial x}+Y \frac{\partial}{\partial y}\right)+Z \frac{\partial}{\partial z}=\left(Y \frac{\partial}{\partial x}-X \frac{\partial}{\partial y}\right) .
$$

Thus, we have

$$
\phi\left(E_{1}\right)=E_{2}, \phi\left(E_{2}\right)=-E_{1} \text { and } \phi\left(E_{3}\right)=0 .
$$


The linearity property of $\phi$ and $g$ yields that

$$
\begin{aligned}
\eta\left(E_{3}\right) & =1, \quad \phi^{2} X=-X+\eta(X) E_{3}, \\
g(\phi X, \phi Y) & =g(X, Y)-\eta(X) \eta(Y),
\end{aligned}
$$

for any vector fields $X, Y$ on $M$.

Moreover, we get

$$
\left[E_{i}, \xi\right]=E_{i}, \quad\left[E_{i}, E_{j}\right]=0, \quad i, j=1,2
$$

Using Koszul's formula, we obtain

$$
\nabla_{E_{i}} E_{i}=-\xi, \quad \nabla_{E_{i}} \xi=E_{i}, \quad i=1,2 .
$$

and others are zero. Thus for $E_{3}=\xi, M(\phi, \xi, \eta, g)$ is a Kenmotsu manifold. Now, the non-zero terms of the semi-symmetric metric connection on $M$ become

$$
\bar{\nabla}_{E_{i}} E_{i}=-2 \xi, \quad \bar{\nabla}_{E_{i}} \xi=2 E_{i} \quad i=1,2 .
$$

With the help of the above results it can be easily verified that

$$
\begin{array}{llll} 
& R\left(E_{1}, E_{2}\right) E_{3}=0, & R\left(E_{2}, E_{3}\right) E_{3}=-E_{2}, & R\left(E_{1}, E_{3}\right) E_{3}=-E_{1}, \\
& R\left(E_{1}, E_{2}\right) E_{2}=-E_{1}, & R\left(E_{2}, E_{3}\right) E_{2}=E_{3}, & R\left(E_{1}, E_{3}\right) E_{2}=0, \\
& R\left(E_{1}, E_{2}\right) E_{1}=E_{2}, & R\left(E_{2}, E_{3}\right) E_{1}=0, & R\left(E_{1}, E_{3}\right) E_{1}=E_{3} . \\
& \text { and } & & \\
& \bar{R}\left(E_{1}, E_{2}\right) E_{3}=0, & \bar{R}\left(E_{2}, E_{3}\right) E_{3}=-2 E_{2}, & \bar{R}\left(E_{1}, E_{3}\right) E_{3}=-2 E_{1}, \\
& \bar{R}\left(E_{1}, E_{2}\right) E_{2}=-4 E_{1}, & \bar{R}\left(E_{2}, E_{3}\right) E_{2}=2 E_{3}, & \bar{R}\left(E_{1}, E_{3}\right) E_{2}=0, \\
\text { (7.5) } & \bar{R}\left(E_{1}, E_{2}\right) E_{1}=4 E_{2}, & \bar{R}\left(E_{2}, E_{3}\right) E_{1}=0, & \bar{R}\left(E_{1}, E_{3}\right) E_{1}=2 E_{3} .
\end{array}
$$

In view of (1.1), one can obtain the torsion tensor $\bar{T}$ with respect to the semisymmetric metric connection as

$$
\begin{array}{r}
\bar{T}\left(E_{i}, E_{i}\right)=0 \text { for } i=1,2,3 ; \\
\bar{T}\left(E_{1}, E_{2}\right)=0, \quad \bar{T}\left(E_{1}, E_{3}\right)=E_{1}, \quad \bar{T}\left(E_{2}, E_{3}\right)=E_{2} .
\end{array}
$$

Since $E_{1}, E_{2}, E_{3}$ forms a basis, the vector fields $X, Y, Z \in \chi(M)$ can be written as

$$
\left(\begin{array}{c}
X \\
Y \\
Z
\end{array}\right)=\left(\begin{array}{lll}
a_{1} & b_{1} & c_{1} \\
a_{2} & b_{2} & c_{2} \\
a_{3} & b_{3} & c_{3}
\end{array}\right)\left(\begin{array}{c}
E_{1} \\
E_{2} \\
E_{3}
\end{array}\right),
$$

where $a_{i}, b_{i}, c_{i} \in \mathbb{R}^{+}$(the set of all positive real numbers), $i=1,2,3$. Using the expressions of the curvature tensors, we find values of the Riemannian curvature and Ricci curvature with respect to the semi-symmetric metric connection as;

$$
\begin{aligned}
\bar{R}(X, Y) Z & =\left[-4\left\{a_{1} b_{2}-b_{1} a_{2}\right\} b_{3}+2\left\{c_{1} a_{2}-a_{1} c_{2}\right\} c_{3}\right] E_{1} \\
& +\left[-4\left\{b_{1} a_{2}-a_{1} b_{2}\right\} a_{3}+2\left\{c_{1} b_{2}-b_{1} c_{2}\right\} c_{3}\right] E_{2} \\
& +\left[-2\left\{c_{1} a_{2}-a_{1} c_{2}\right\} a_{3}-2\left\{c_{1} b_{2}-b_{1} c_{2}\right\} b_{3}\right] E_{3} \\
\bar{S}\left(E_{1}, E_{1}\right) & =\bar{S}\left(E_{2}, E_{2}\right)=-6, \bar{S}\left(E_{3}, E_{3}\right)=-4 .
\end{aligned}
$$


In view of the expression of the endomorphism $\left(E_{i} \wedge_{g} E_{j}\right) E_{w}=g\left(E_{j}, E_{w}\right) E_{i}-$ $g\left(E_{i}, E_{w}\right) E_{j}$ for $1 \leq i, j, w \leq 3$ and equations (7.5) and (7.8), one can easily verify that

$$
\begin{aligned}
\bar{S}\left(\bar{R}\left(E_{i}, E_{3}\right) E_{j}, E_{3}\right)+\bar{S}\left(E_{j}, \bar{R}\left(E_{i}, E_{3}\right) E_{3}\right) & =-2\left[\bar{S}\left(\left(E_{i} \wedge_{g} E_{3}\right) E_{j}, E_{3}\right)\right. \\
& \left.+\bar{S}\left(E_{j},\left(E_{i} \wedge_{g} E_{3}\right) E_{3}\right)\right],
\end{aligned}
$$

in view of the above equation Theorem 4.1. is verified.

\section{R E F E R E N C E S}

1. Ajit Barman and U. C. De: Projective curvature tensor of a semi-symmetric metric connection in a Kenmotsu manifold. International Electronic Journal of Geometry. 6 (2013), 159-169.

2. K. AmUR and S. S. PUJAR: On submanifolds of a Riemannian manifold admitting a metric semi-symmetric connection. Tensor, N. S. 32 (1978), 35-38.

3. C. S. BAGEWADI: On totally real submanifolds of a Kahlerian manifold admitting Semi symmetric metric F-connection. Indian. J. Pure. Appl. Math. 13 (1982), 528-536.

4. C. S. Bagewadi, D. G. Prakasha and Venkatesha: Projective curvature tensor on a Kenmotsu manifold with respect to semi-symmetric metric connection. Seria. Mathematica 17 (2007), 21-32.

5. A. BARMAN: On para-Sasakian manifolds admitting semi-symmetric metric connection. Publ. De L'Institut Math. 109 (2014), 239-247.

6. T. Q. BinH: On semi-symmetric connection. Periodica Math. Hungerica, 21 (1990), 101-107.

7. D. E. BlaIR: Contact manifolds in Riemannian geometry. Lecture Notes in Mathematics, Springer-Verlag, Berlin, 1976.

8. M. C. CHAKI and B. CHAKI: On pseudosymmetric manifolds admitting a type of semisymmetric connection. Soochow J. Math. 13 (1987), 1-7.

9. U. C. DE: On a type of semi-symmetric connection on a Riemannian manifold. Indian J. Pure Appl. Math. 21 (1990), 334-338.

10. R. Deszcz: On pseudosymmetric spaces. Bull. Soc. Math. Belg., Ser. 44 (1992), 1-34.

11. A. Friedmann and J. A. Schouten: Über die Geometric der halbsymmetrischen Übertragung. Math. Zeitschr 21 (1924), 211-223.

12. H. A. Hayden: Subspaces of a space with torsion. Proc. London Math. Soc. 34 (1932), 27-50.

13. S. K. HUI and RiCHARD S. LEMENCE: Ricci pseudosymmetric generalized quasiEinstein manifolds. SUT J.Math. 51 (2015), 195-213.

14. K. Kenmotsu: A class of almost contact Riemannian manifolds. Tohoku Math. J. 24 (1972), 93-103.

15. D. G. Prakasha, Aysel Turgut Vanli and C. S. Bagewadi: Some classes of Kenmotsu manifolds with respect to semi-symmetric metric connection. Acta Mathematica Sinica. 29 (2013), 1311-1322. 
16. R. N. Singh, S. K. Pandey, G. Pandey and K. Tiwari: On a semi-symmetric metric connection in an $\epsilon$-Kenmotsu manifold. Commun. Korean Math. Soc., 29 (2014), No.2, 331-343.

17. Venkatesha, K. T. Pradeep Kumar and C. S. Bagewadi and Gurupadavya INGALAHALLI: On concircular $\phi$-recurrent $K$-contact manifold admitting semisymmetric metric connection. Hin. Publ. Corp. Intl. J. Math. Sci. 2012 (2012).

18. K. YAno: On semi-symmetric metric connections. Rev. Roumaine Math. Pures Appl. 15 (1970), 1579-1586.

Venkatesha Venkatesh

Associate Professor

Department of Mathematics, Kuvempu University,

Shankaraghatta-577 451, Karnataka, INDIA

vensmath@gmail.com

Arasaiah Arasaiah

Department of Mathematics, Kuvempu University

Shankaraghatta-577 451, Karnataka, INDIA

ars.gnr94@gmail.com

Vishnuvardhana Srivaishnava Vasudeva

Department of Mathematics, GITAM School of Technology

GITAM(Deemed to be university), Bangalore, Karnataka, INDIA

svvishnuvardhana@gmail.com

Naveen Kumar Rahuthanahalli Thimmegowda

Department of Mathematics, Siddaganga Institute of Technology

B H Road, Tumakuru-572 103, Karnataka, INDIA.

rtnaveenkumar@gmail.com 